be said of the humans to the lower side of line " $\mathrm{A}$ " with respect to their competitiveness with the top machines, as they lost by a score of $13.5-2.5$.

All this is very interesting, and is no doubt due to the fact that the organizers wished to get a representative sample of players. However, attempting to stratify the field is very instructive.

Clearly, the top 6 computers could do very well against any but well-versed IM-strength players. First of all, one should congratulate Fidelity Mach IV+ on its outstanding performance. This would be awesome if it were not for the fact that this same type of machine lost to both Deep Thought and Hitech in the World Computer Championships just following. However, this machine played only two of the top 4 humans, but managed an even score. No other machine did nearly as well, although it could be said that its point in the last round against Ligterink was due to the latter trying too hard to win in an attempt to share first place. Nevertheless this was a sterling performance, especially given how well the top 4 humans did otherwise.

So the top 4 humans outscored the top six machines by 12.5 to 1.5 , the only machine points coming from two Fidelity products. Why did the machines do so badly against this group? The answer, to this analyst, is that these players were well prepared and played accurately tactically when necessary. It was not unusual to see a human start the game $1 . \mathrm{Nc} 3 \mathrm{~d} 52$. e4 d4 3. Nce2 and now White has a nice strategic game to play and Black has to invent what to do, which is usually hard for the machines. Only Mach IV (as Black vs. Van den Berg) made much of a fight out of it. Generally, the humans just kept the position simple and steered the game into favorable strategic situations. They demonstrated that this could be done in many ways. I was amazed at how meekly most of these top machines acceded to their opponent's wishes. The humans to the lower side of line "A" apparently were not able to do this.

While the above is a tip of the hat to the top 4 humans, it must be noted that they are not presently considered among the top players in the world. Thus, it would be easy to imagine that those GMs who are now so worried about computer encroachment could learn a lot by a perusal of the games played to the upper side of line "A". (Although it must be said they are principally worried about Deep Thought, and DT did not participate here.)

Finally, I must congratulate Mr. Bakker on his courage and good sportsmanship in coming out to play the monsters. It had been just about some 4 years (December, 1985), since his rather premature prediction that "He would have nothing to fear from computers in the next 5 years", based on a single win versus Cray Blitz [ICCA Journal, Vol. 8, No. 4, p. 280]. He played only one of the top six, but did not severely dominate the rest, so I believe one can safely say that he did "have something to fear" and was well advised to turn away my challenge to back up his statement with some money. I hope that next year's event will produce a better showing by the top machines.

\title{
THE VALVO-DEEP THOUGHT UNIX MAIL MATCH
}

\author{
Mike Valvo
}

Towards the end of November, 1988, I proposed a correspondence match between Deep Thought and myself to be conducted over the Netnews facility of the UNIX System. This computer mail system is similar to the LINC except it does not support interactive communication. It contains a newsgroup known as rec.games.chess where it is possible to post an article for all the members of the group to read. Each member is free to post a reply to any article.

The activity on the net was not particularily interesting, so I thought it might be fun to play a game against the computer and post my comments move by move. The computer would not be able to read what I said and everyone could vicariously participate as the game went along.

I had no idea what the results would be. Deep Thought was fresh with victory from the Software Tools tournament and I was near the top of my form in recent years. Deep Thought's USCF rating at the time was 2551 and mine was 2488 . 
It turned out that this time rate (one move every three days) was more in my favor than the computer's. The faster the time rate, the better it is for computers and the slower, the better it is for humans. The amount of time required to look an additional ply (a half move) is so much that computers do not benefit very much from the additional time. The best that can be done is to have a strong human helping the computer with different lines to try. At the current time I am playing a consulting match teamed up with former World Computer Champion, Belle, in this manner.

These games brought into focus the strengths and weaknesses of computers. Their surprising strength is defending losing positions. The computer exhibited incredible patience and ceded ground slowly. Another surprise was the success of positional material sacrifices. The machine, not having positional judgement, is susceptible to such ploys and in my opinion this is an important factor in playing them.

Anyway, on to the two games!

\author{
Valvo-Deep Thought \\ UNIX Mail Match (Game 1) 11/88-3/89 \\ Alekhine's Defense
}

1. e4 Nf6 2. e5 Nd5 3. d4 d6 4. c4 Nb6 5. f4 dxe5 6. fxe5 Nc6 7. Be3 Bf5 8. Nc3 e6 9. Nf3 Bg4 10. Be2

The modern continuation is 10 . Qd2, but that does not suit my style. Black can then play $10 . . . \mathrm{Bb} 4$ ! 11 . Be2 (11. 0-0-0 Na4 or 11. a3 Be7 12. Ne4 a5 13. Be2 Bf3 14. gf3 Bh4 and Black is better) Bf3 12. gf3 Na5 13. b3 c5 14. dc5 Nd7 15. 0-0-0 Qe7 16. Kb2 Nc5 17. Bg5 Qc7 18. Qe3 a6 19. Rd6 Nd7=.

10. ... Bxf3 11. gxf3 Qh4+ 12. Bf2 Qf4 13. c5

My game against Alburt in Philadelphia, 1988 continued 13. Qc1 Qxc1+ 14. Rxc1 0-0-0 15. Rd1 g6?! (15. ... Bb4 $\Rightarrow$ 16. a3 Bh6 17. b4 Rd7 18. Ne4 Rhd8 19. c5 Nd5 20. b5 Nce7 21. c6! bxc6 22. bxc6!? (22. Nc5!) Nxc6 23. Bb5 Nde7?? (23. ... Nb8) 24. Ba6+ 1-0 (24. ... Kb8 25. Rb1+-).

13. ... Nd7!

Not 13.... Nd5 14. Nxd5 exd5 15. Qd2 +=.

14. Qc1 Qf5 15. Qb1 Qxb1+

The dance is over as 15. ... Qf4?! runs into 16. Qe4.

16. Rxb1 0-0-0 17. f4 Be7 (see Diagram 1)

The Deep Thought team announced that, with this move, the computer was out of book.

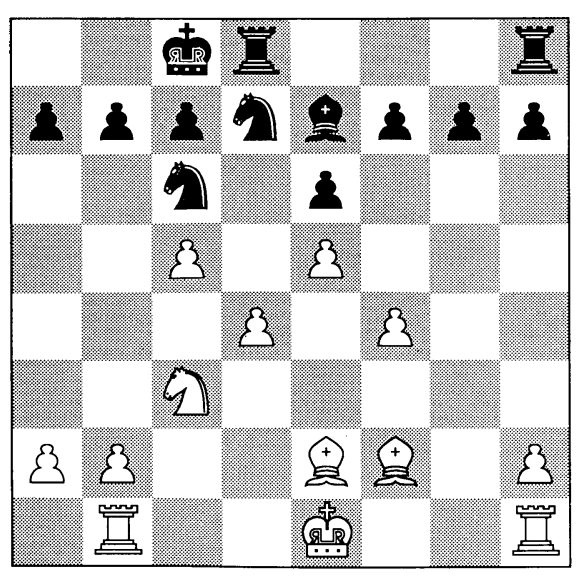

\title{
18. Rd1 g5
}

This is an' interesting idea. If $18 . . . \mathrm{Nf} 8$, Black has to figure out how to deal with 19. d5 exd5 20. Nxd5 intending 21. b4.

19. fxg5 Bxg5 20. Bf3!?

I felt like a wimp making this move. I lulled myself into thinking that the computer would never allow Bc6 doubling its Pawns. Better was 20. Ne4! Be7 (20.... Bf4 21. a3! Rhg8 22. b4 Ne7 23. Bh4 Rde8 24. Nf6) 21. a3!

\section{DIAGRAM 1}

Position after 17.... Be7

20. ... f5?

This move ends the strategical portion of the game. From now until the end, Black can only defend. The possibility of $\mathrm{f} 6$ was Black's main counterplay idea. After f5, the black position drifts into passivity. 
Other black possibilities are 20. ... f6 21. Ne4 Rhg8 22. h4 Bf4 23. exf6 Rdf8 with unclear chances. Interesting is 20. ... Ne7 21. b4 c6 22. b5 Nd5 23. Ne2! (23. ... cb5?! 24. Bd5 ed5 25. Nc3).

21. $0-0$ !?

This is perhaps too greedy. The King is safe enough in the center and better was $21 . \mathrm{Ke} 2$ !

21.... Nb4 22. Rfe1!

Not 22. a3? Nc2! followed by Ne3.

22. ... Rhg8 23. Kh1 c6 24. a3 Na6? 25. b4 Nc7 26. a4 a6 (see Diagram 2)

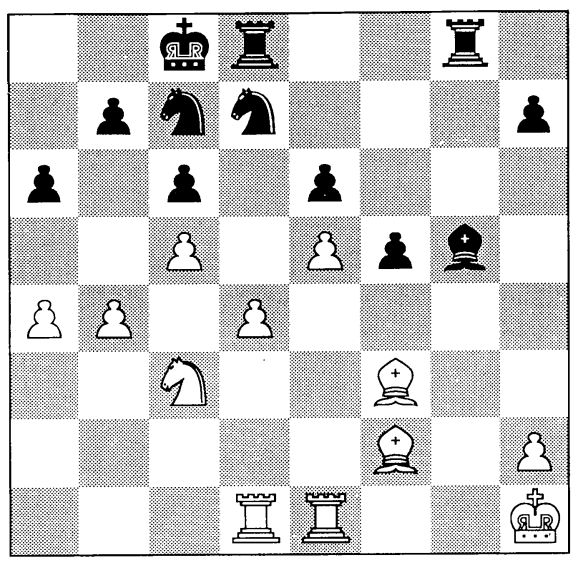

27. Re2!

The position still contains dangers for White: 27 . b5?! axb5 28. axb5 cxb5 29. Rb1? Nxc5!

27. ... Be7 28. Rb2! Nd5 29. Nxd5 cxd5 30. b5 axb5 31. axb5 Rg7 32. Ra1 Nb8 33. Rba2!

White will not allow Black a whisp of counterplay. One Rook on the first rank will prevent any tricks like 33. Ra8? Rdg8 intending $\mathrm{Bh} 4$ !

33. ... Rdg8 34. Ra8 Bg5 35. b6

White has decided that the final blow will come as a result of an eventual $\mathrm{Ba} 6$ or Pawn c6.

\section{DIAGRAM 2}

Position after 26. ... a6

\section{5. ... Bd8 36. Bh5}

Putting Black on notice regarding his weak $\mathrm{f7}$ point. I later wondered, though, if 36 . Be3!? with the idea of Bf4, $\mathrm{Bd} 5$ !?, and e6 threatening the b8 Knight might be more effective. If 36. ... f4 37. Bf2!+- intending Rf1, Be1-a5, $\mathrm{Rc} 1$ and $\mathrm{c} 6$.

36. ... Rf8 37. Be2

If now 37. Be3?!, then 37. ... f4 38. Rf1 Rg5 39. Be2 Rgf5 40. Bf2 f3. Also interesting is 37 . Rf1 to try to lure the $\mathrm{f} 4$ forward one square. Then White will blockade it and $\mathrm{Rc1}$ will not get harassed by $\mathrm{Bg} 5$.

37. ... Rfg8 38. Be3 h5 (see Diagram 3)

This is a sound pawn sacrifice. Not instead 38. ... Rg2? 39. Bh5 R8g7 (39. ... R2g7 40. Bh6+-) 40. Bf7!+-.

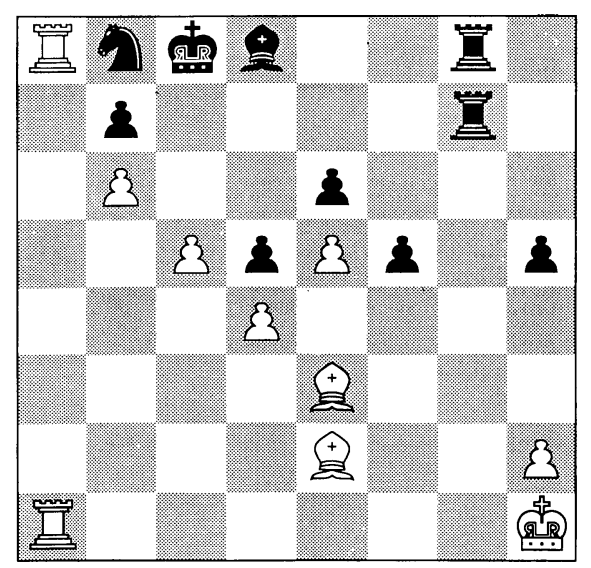

\section{Rb1!}

White ignores the Kingside distractions and prepares 40. 66 bc6 41. Ba6. Bad is 39. Bxh5? Rh7 40. Be2 (40. Bf3? Rg3 41. Rf1 Rhh3) Rgh8 41. Bf4 Bg5! 42. Bxg5 Rxh2+ 43. Kg1 Rxe2 44. Be7 Rhh2=.

39. ... Be7!

The best. White's threat is apparent after 39. ... Bh4? 40. c6 bxc6 41. Rba1 Kb7 42. R1a7+ Kxb6 43. Rxg7+-. 


\title{
40. Bb5!
}

The c6 idea no longer works because the e7 Bishop shields the seventh rank: 40. c6? bxc6 41. Ba6+ Kd7-+. 40 . $\mathrm{Bb} 5$ sets up ideas like $\mathrm{c} 6$ and the possibility of a rook sacrifice on $\mathrm{b} 8$ to achieve Ra8 mate.

40.... Bd8 (see Diagram 4)

This idea of keeping an eye on the b6 Pawn is' virtually forced. Otherwise if $40 . . . \mathrm{Bg} 5$ 41. Bxg5 Rxg5 42. c6 bxc6 43. Bxc6+- and Rba1, Rb8 and Ra8 mate soon follows. If 40. ... Rg2 41. Ba6 bxa6 42. c6 Bd8 43. c7 Bxc7 44. bxc7 Kxc7 45. Raxb8+-.

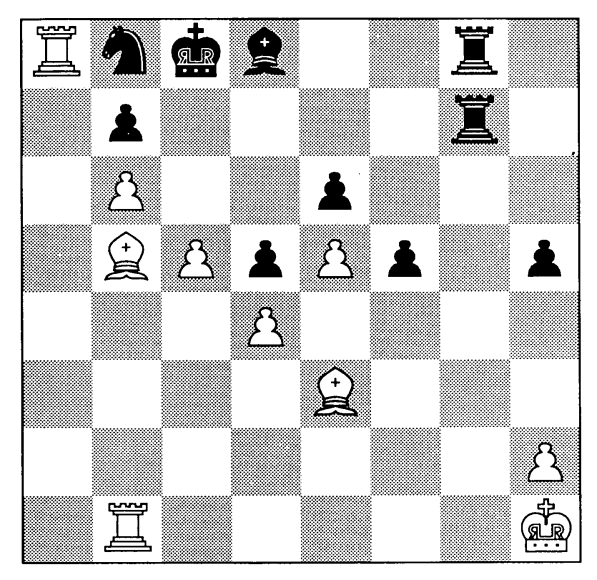

\author{
41. Ba4! \\ To protect b6 so $c 6$ is a threat. \\ 41.... f4
}

Zugzwang looms. If $41 . \ldots \operatorname{Rg} 2 ?$ 42. Bd7+! $\operatorname{Kxd7} 43 . \operatorname{Rxb8}$ Kc6 44. Rxd8+-. If 41. ... Rg4 42. c6 bxc6 43. Bxc6 f4 44. Bf2 Rg2 45. b7+ Kc7 46. Rc1 Rxf2 47. Bxd5+ Kd7 48. Rxb8 exd5 49. Rbc8 Rgg2 50. Rxd8+ Ke6 51. Rd6+ Kf5 52. Rf6+ Ke4 53. Rg6!+- .

\section{DIAGRAM 4}

Position after 40. ... Bd8

\section{2. ... Bxf4 Rf7!?}

This allows a crippling bishop move. Better is $42 \ldots$... Rg4 43. Be3 h4.

43. Bh6! h4 (see Diagram 5)

Black's alternatives are not more promising. For example, 43. ... Rf2 44. Bd7+ Kxd7 45. Rxb8 Kc6 46. Ra1!! h4 (46. ... Rgg2?! 47. Bf4!+- or 46. ... Rb2!? 47. Rf1 Rgg2 48. Bf4 Rgf2 49. Rxf2 Rxf2 50. Bg3 Rf8 51. Bh4 Kd7 52. Bxd8 Rxd8 53. Rxb7+-) 47. Rc8+ Kd7 (47. ... Kb5 48. Rb1+ Kc4 49. c6+-).

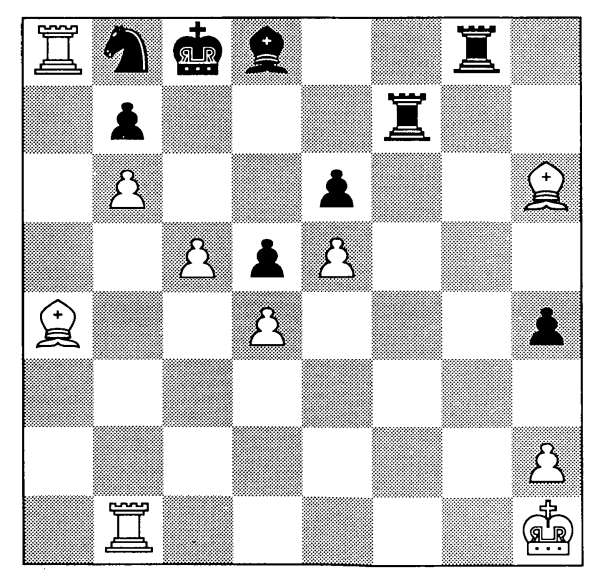

DIAGRAM 5

Position after 43.... h4
48. c6+! bxc6 (48. ... Kxc8 49. Ra8 mate) 49. b7+-. Black's helplessness becomes clear after 43. ... Rh7? 44. Rf1! Rxh6?? 45. Rf7+- or 43. ... Rh8? 44. Rg1! Rxh6?? 45. Rg8+- intending 46. $\mathrm{Rb} 8$ and 47. $\mathrm{Rd} 8$ mate.

\section{Bb5}

A final squeeze. Again if 44.... Rh7 45. Rf1! or 44.... Rh8 45. Rg1! The direct 44. $\operatorname{Rg} 1$ should also win: 44. Rg1!? Rxg1+ 45. Kxg1 Bg5 46. Bxg5 Rg7+-. 


\title{
44. ... Be7
}

There are no more defenses: 44. ... h3!? 45. Rg1 Rxg1+ 46. Kxg1 Rf3 47. Be2!+-.

45. c6 bxc6 46. Bxc6 Rf3 47. Rba1!

Going for the direct mate: 48 . Rb8 Kb8 49. Ra8 mate.

47.... Ba3 48. Bd2 Resigns 1-0

For if 48. ... Rb3 49. Ba5 (Deep Thought). White's threat is 49. Bb4!

\author{
Deep Thought-Valvo \\ UNIX Mail Match (Game 2) 12/88-3/89 \\ King's Gambit Declined: Falkbeer Counter Gambit
}

1. e4

I had seen Deep Thought's play against various IMs and had no idea how to play against 1. e4 by this tactical monster. As a result, I decided to play solidly with 1.... e5. Little did I realize what was in store!

1. ... e5 2. f4

This I did not expect: a King's Gambit. I 'thought' Deep Thought played the Ruy Lopez. Well, so much for thinking. I knew its book could not be current, so it made sense to whip out the topical 2 ... d5 3 ed5 c6!?

2. ... d5 3. exd5 c6 4. Nc3 exf4 5. Nf3 Bd6 6. d4 Ne7 7. dxc6 Nbxc6 8. d5

White's best is probably 8. Bc4 0-0 9. 0-0 Bg4 10. Ne4 Bc7 11. c3 as played in Illescas-Nunn, Dubai Olympiad 1986. But better than 11. ... Nd5?! 12. Nc5! Rb8 13. Qe1 Re8 (13. ... g5!?) 14. Qh4! with advantage, is 11. ... $\mathrm{Ng6}=$. Nunn has played this variation since and one has to assume he has some improvement in mind.

8. ... Nb4 9. Bc4 0-0

I think this is best, but it is virtually ignored in practice. Usually played is 9. ... Bf5!? $10 . \mathrm{Bb} 3$ and Black has gone two ways:

10. ... Qb6! 11. a3 Na6 12. Qd4 Nc5 13. 0-0 0-0 14. Kh1 Bd3! and Black was somewhat better in Gallagher-Nunn, London, 1987.

10 ... 0-0 11. 0-0 Bg4 12. Ne4 with a very small edge in Spassky-Polgar, Z., Wellington, 1988.

10. a3 b5!!

Prior to the discovery of this resource, Black played 10... Na6 11. 0-0 Bg4 12. Kh1 with a small white edge as in Hebden-Henley, New York 1983.

11. Bb3

This is the least exciting of the three white alternatives. The others, however, pose no problems for Black:

11. Bxb5 Nbxd5 12. Nxd5 Nxd5 13. Bc6 Ba6! 14. Qxd5 Qe7+! (Elliott Winslow; not 14.... Re8+? 15. Kd1 Be2+ 16. Kd2 Bc4 17. Qd4! [17. Bxa8?? Bxd5 18. Bxd5 Bb4+ 0-1 Hoyos Milan-Garcia, Guid., Columbia, 1987] 17. ... Be5 18. Re1!!+- winning even though 18. ... Qd4 19. Nd4 Rad8 clouds the issue) 15. Qe4 Qxe4+ 16. Bxe4 Rfe8 with all the chances.

11. Nxb5 Nbxd5 12. Bxd5 Nxd5 13. c4 Re8+ 14. Kf1 Bc5!! (Deep Thought) with the following continuations given by Berliner/Hitech: 15. Qxd5 (15. cxd5 Ba6 16. a4 Qa5-+ if now 17. Qd3 Rab8!) 15. ... Bf5!-+.

11. ... Na6 12. Nxb5 Qa5+ 13. Nc3 Nc5 14. Ba2

If White considers himself in serious trouble, he could opt to castle right now: 14. 0-0 Nxb3 15. cxb3 Bb7 (also possible is 15 . ... Ba6!? 16. Re1 Rfe8 intending to meet $17 . \mathrm{Ng} 5$ !? with 17 . ... Nd5!) $16 . \mathrm{Ng} 5 \mathrm{Nxd5} 17 . \mathrm{Nxd5}$ Qxd5 18. Qxd5 Bxd5 19. Bxf4 and now 19. ... Bc5! (Speelman) with a very small black edge.

14. ... Ba6!! (see Diagram 6) 


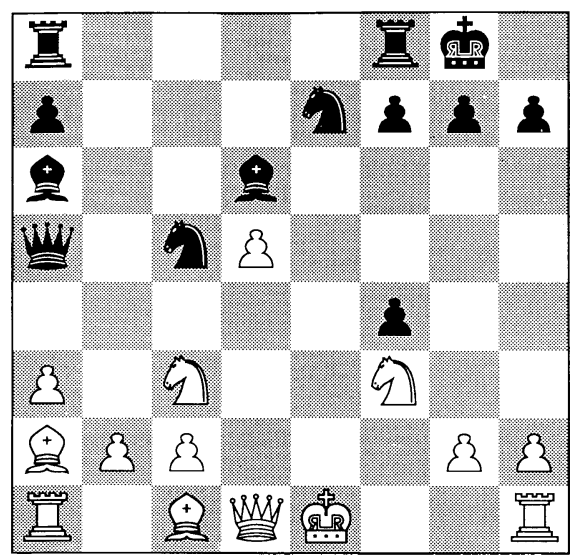

At the time I thought this was the best chance as it seemed my intended 14. ... Ne4!? would run into 15 . Qd4 Nc3 16. Bd2. However, 16. ... Re8! is then more than adequate. White's best is $15 . \mathrm{Bd} 2 \mathrm{Nxd} 216 . \mathrm{Qxd} 2 \mathrm{Rb} 817 . \mathrm{Ne} 4$ ! with an unclear position.

When the ideas behind 14. ... Ba6 began to flow for me, I 'knew' it was the move I wanted to make. The only other alternative was 14 ... Ne4 and I was under the impression at the time that 15 . Qd4 refuted it. The more I looked at 14. ... Ba6, the better it felt, so I never returned to recheck 14. ... Ne4. Still, it was not without a great deal of trepidation that I mailed off this piece sacrifice.

\section{DIAGRAM 6}

Position after 14. ... Ba6

Ken Thompson visited the Deep Thought team in Pittsburgh at this point and indicated that things were in near panic after reception of Black's fourteenth. I believe he said at the time Deep Thought searched 35 plies (17 moves!) and could not find a refutation.

This caused a stir on the net and a Deep Thought groupie wanted to bet me five dollars that the machine could hold its position. Everything quickly quieted down when a member of the Deep Thought team told of the difficulties the machine was having.

\section{5. b4 Qc7 16. bxc5}

Deep Thought looked at 16 . b5 and considered it nearly as good as the test. Black could then play the quiet 16 . ... Bb7 or the sharp $16 . . . \mathrm{Bb} 5$ !

16. ... Rfe8! 17. Ne2 Qxc5 18. c4 Nxd5! 19. Qd4!

I think this is best. If 19. Qxd5 Rxe2+ 20. Kd1 Qxd5+21. cxd5 Rxg2 and Black has the idea g5-g4 in mind. I thought the machine would go for this line because it would remain a piece up.

19. ... Qxd4! 20. Nfxd4 Bc5! (see Diagram 7)

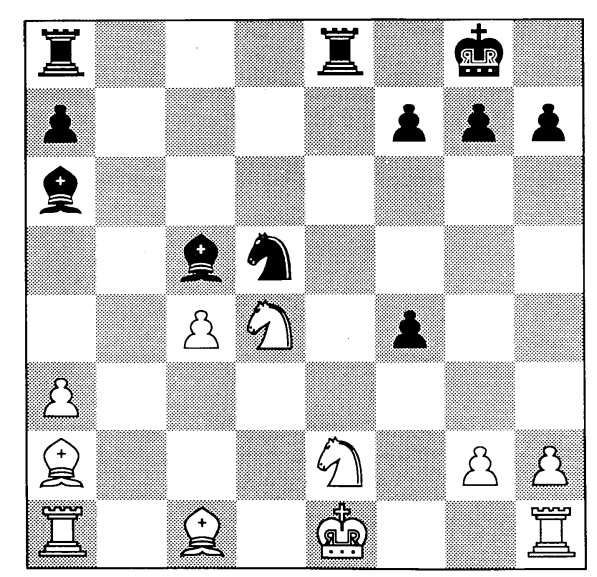

DIAGRAM 7

Position after 20. ... Bc5
This was not an easy move to see and I had to consider it before playing 14. ... Ba6. Interesting is the idea 20. ... Nc3?!, but it just doesn't work. After the text, however, it becomes a threat.

\section{Kd2}

This was totally unexpected. I thought I would meet 21 . Kd1 and I had intended to play 21 ... Ne3. The try 21 . Kf1 is easily dealt with by 21 .... Re4, but the similar 21 . Kf2 Re4 (21. ... $\mathrm{Nc3}$ !?) would have led to complicated play. An example of the possibilities is 21. Kf2 Re4 22. Kf3 Rae8 23. cd5 Re2 24. Ne2?? Be2 25. Kf4 Bd6 26. Kf5 Re5. 
Fascinating is the idea 21. Bb2. 21... Rab8 can be met by 22. 0-0-0!? and White threatens the black Knight on d5. My first instinct was 21... f3!? (21... Ne3) intending 22. gf3 Bd4 23. Bd4 Nf4, but 22. cd5! complicates things immensely. An interesting continuation is 21 . Bb2 f3 22. cd5 Rab8 23. Bc3 Bd4 24. Bd4 Re2 (24. ... $\mathrm{Be} 2$ !?) 25. Kd1 Ra2 26. Ra2 Rb1 27. Kd2 Rh1 28. d6! with at least equality.

I still think 21. Kd1 was best: 21. ... Ne3 22. Be3 Re3 23. Nc2 Rd8 24. Ke1 Red3! and now

(1) 25. Nf4 Rd2 26. Nb4 Bf2! 27. Kf1 Bd4 28. Rb1 Re8-+

(2) 25. Nb4 Ra3 26. Na6 Ra6 and White is all pinned up

(3) 25. Rf1! g5 26. h4 h6 27. hg5 hg5 28. Rh1 unclear.

21. ... Ne3 22. Kc3 Rac8! 23. Bb2?!

I expected 23. $\mathrm{Nxf} 4$ !? Bxd4+ 24. Kxd4 Nc2+25. Kc3 Nxa1 26. Kb2! and it is still anyone's game. The smoke has cleared and Black has a Rook and a Pawn for the two pieces.

The refutation of 23. Nxf4 is the dynamic 23. ... Re4! (Henley) and after either 24. Nfe2 or 24. Nde2, 24. ... $\mathrm{Nc} 4$ ! and the white King is caught in a deadly cross fire.

The Deep Thought operator mentioned on the next move something about sending off the 23rd move too quickly when he sent Deep Thought's 24th. Apparently, the machine was still considering its reply and he misread the information. I offered to let them retry their 23rd, but they declined. Even today, I do not know what Deep Thought intended in this position.

23. ... Nxg2

Deep Thought considered this a significant mistake and thought that 23 ... Bb6! would have led to a large black edge. The human part in me, however, cried for some tangible compensation for the piece it gave up some nine moves earlier. I did not give enough weight to the 23. ... Bb6 and 24. ... Ba5+ idea. I just saw that 23... $\mathrm{Ng} 2$ would maintain an edge in initiative and recoups some material.

24. Raf1 Red8 25. Rhg1 Re3+ 26. Kd2 f3 27. Rxf3 Rxf3 28. Rxg2 Rh3!

I wanted to avoid any dangers such as 28. ... Bxa3? 29. Kc2! Rh3 30. Nf4 Rh4 31. Rxg7+ Kxg7 32. Nf5+ Kg8 33. Nxh4 Bxb2 34. Kxb2 Rd2+ 35. Ka3 Rxh2 36. Nf5 Rf2 37. Nd5 and if anything, White is for choice.

\section{Kc1 g6!}

Why take risks? What can White do with this tempo?

30. a4 Bb7 31. Rf2 Ba8! (see Diagram 8)

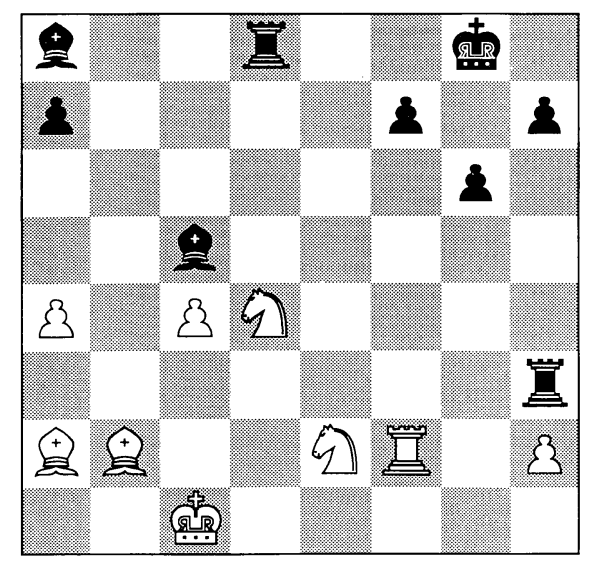

DIAGRAM 8

Position after 31.... Ba8
Black tries to take advantage of the fact that White is nearly without moves. The only moves that seem available to White are 32. a5 and 32. Bb1. The former can be dealt with by losing a move through 32 ... f5 and the latter by swinging the Rook on $\mathrm{d} 8$ to the b-file threatening $\mathrm{Rb} 4$.

Direct attempts such as 31. ... Rh4!? 32. Kd2 Bxd4 33. Bxd4 Rhxd4+ 34. Nxd4 Rxd4+ 35. Kc3 and 31. ... Be4!? 32. Bb1! Bb1 33. Kb1 Rh4 34. Kc2 Rhd4 35. Nd4 Bd4 36. Bd4 Rd4 $37 . \mathrm{Kc} 3$ both give White some drawing hopes. 
32. Bb1 Rb8 33. Ba2 Rd3 34. Rf4 Rd2 35. Kxd2 Rxb2+ 36. Nc2 Rxa2 37. Nc3 Rb2 38. Rf6 Kg7 39. Rf1

Deep Thought changes its mind about 39. Ra6. The text, however, reduces White to near passivity and perhaps 39. Ra6!? should have been tried. Black has 39. ... Bb6 (threatening to trap the Rook with 40 ... Bb7) 40. a5 $\mathrm{Bg} 1$ 41. h4 Rb3 and while Black has ideas of going after White's weak h-Pawn, White has a modicum of play.

39. ... f5 40. Nd5 Bxd5 41. cxd5 Rb3 42. h4 Kf6 43. Re1

(43.... h5 Ke5!)

Rh3 44. Re6+ Kf7 45. a5

(45. ... Rc6 Bb6 is completely hopeless)

45. ... Rxh4 46. Rc6 Bb4+ 47. Nxb4 Rxb4 48. Rc7+ Kf6 0-1

After 49. Rh7 Rd4 50. Kc3 Rd5 51. Ra7 g5 the win is elementary. The only reasonable try is 49. Kc3!?, but the Deep Thought team checked out all the possibilities with a local expert and decided it was not worth playing on.

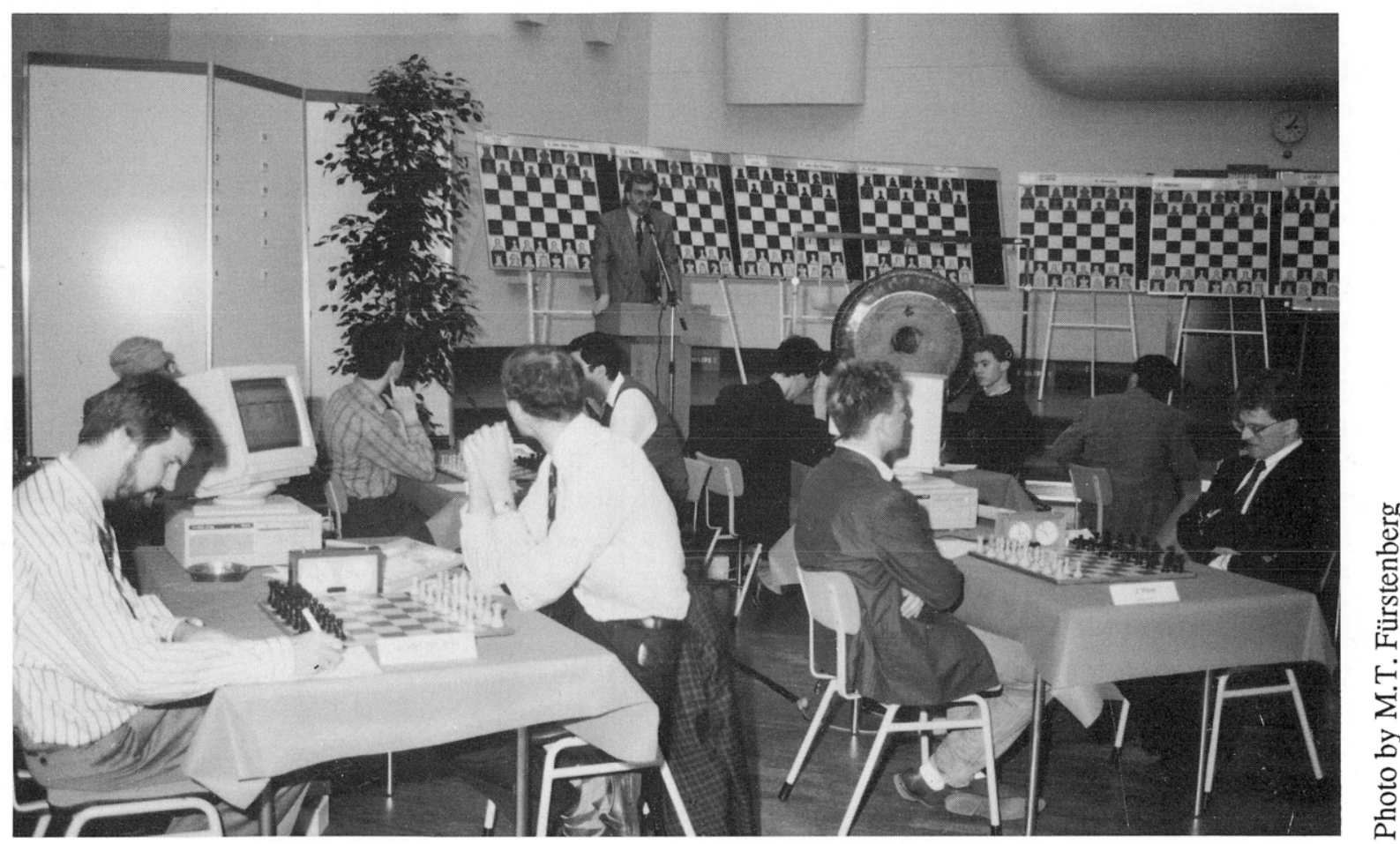

Chesstersely in Dutch.

In the front from left to right: IGM John van der Wiel, Tom Pronk (operator ChipTest),

IGM Jeroen Piket and Maarten van der Meulen (operator Hitech);

behind the microphone IM Hans Böhm.

AVRO/PHILIPS Dutch Championship 1989, Hilversum, The Netherlands. 